\title{
Isolation and Antifungal Effects of Plants Extracts on Malassezia species Isolated from Scalps of Primary School Pupils and Bingham University Students
}

\author{
Maikenti James Ishaku ${ }^{1^{*}}$, Egah Ruth Grace $^{2}$, Adogo Lillian Yami $^{2}$ \\ and Koggie Amos Zamfara ${ }^{2}$ \\ ${ }^{1}$ Department of Zoology, Federal University of Lafia, Nasarawa State, Nigeria. \\ ${ }^{2}$ Department of Biological Sciences, Bingham University, Nigeria.
}

\begin{abstract}
Authors' contributions
This work was carried out in collaboration among all authors. Author MJl designed the methodology and supervised the study. Authors KAZ and ERG managed the analysis of the study and the literature of the study. Author ALY prepared the first draft of the manuscripts. All authors read and approved the final manuscript.

Article Information

DOI: $10.9734 / A R R B / 2021 / \mathrm{v} 36 \mathrm{i} 130331$ Editor(s):

(1) Dr. Md. Torequl Islam, Federal University of Piaui, Brazil.

Reviewers:

(1) Faisal Hammad Mekky Koua, Deutches Elektronen-Synchrotron, Germany. (2) Derry K. Mercer, Nova Biotics Ltd., United Kingdom. Complete Peer review History: http://www.sdiarticle4.com/review-history/65464
\end{abstract}

Original Research Article

Received 12 December 2020

Accepted 18 February 2021

Published 05 March 2021

\section{ABSTRACT}

Aim: The aim of the research was to evaluate the antifungal activities of the leaf extracts of Senna alata and Lawsonia inermis on Malassezia spp. isolated from the scalps of primary school pupils and Bingham University students.

Place and Duration of the Study: This study was carried out in the department of Biological sciences, Bingham University Karu Nasarawa state between July to September, 2017.

Methodology: The plant species Senna alata and Lawsonia inermis was collected from Garki district of the Federal Capital Territory Abuja and Sayina village of Auta-balefi Karu Local Government Area of Nasarawa state respectively. These plant species were identified by plant experts in the Department of Biological Sciences, Bingham University, using characteristic features of the leaves such as the shapes, sizes and flower corresponding to the herbarium specimens. 
Other features included plant height, fruits type, nature of the branches, colour of the stems, present or absence of spines, and number of flowers etc. Senna alata and Lawsonia inermis plants extracts were obtained by maceration in $80 \%$ ethanol, soaking and boiling of the powdered leaves of the plants respectively. A total of 83 dandruff scrapings were collected from both the pupils of Kodope primary school and Bingham University students. The identification of Malassezia species was made microscopically by methylene blue staining and wet mounting. Each slide was examined under high power field microscope (40X objective) to detect the presence of hyphae and budding yeast cells. The positive samples were cultured on Sabouraud Dextrose Agar (SDA) plates and incubated at $37^{\circ} \mathrm{C}$ for 3-5 days. Fungal growth was observed. Gram's staining procedure and biochemical tests such as catalase test, bile Esculin test and Tween assimilation test using Tween 20 and 80 was carried out. The evaluation of the antifungal effects of Senna alata and Lawsonia inermis on Malassezia sp. was carried out using the well diffusion method.

Results: Out of 83 samples analyzed, 21 were positive for Malassezia spp. Three (3) species were identified and isolated; namely Malassezia restricta, Malassezia globosa and Malassezia furfur.

The antifungal effects of the plant extracts revealed that the Malassezia species were susceptible to the ethanolic extract of Senna alata with a mean zone of inhibition $11.00 \pm 0.969$. The Aqueous extracts of Senna alata showed significant antifungal effect on the fungi with mean zone of inhibition $10.00 \pm 0.969$ when compared to the control, while the boiled extract had no effect. The ethanolic extract of Lawsonia inermis was the only extract recorded to have antifungal effect on the fungi, with mean zone of inhibition $7.71 \pm 0.876$.

The relationship between of Malassezia infection with age, gender, and institutions was determined by Chi-square test through the use of statistical package for social science (SPSS). There was no significant difference in Malassezia infection between the ages ( $P=0.189)$ as well as gender $(\mathrm{P}=0.367)$. However, the distribution of infection between the institutions shows a significant difference $(p=0.043)$.

Conclusion: This study reveals that naturally acquired herbs of $S$. alata especially and $L$. inermis may be effective in combating Malassezia species which are the causative agents of dandruff and hence, the use of naturally acquired herbs should be encouraged in combating dandruff.

Keywords: Fungi; Malassezia spp.; dandruff; antifungal; locally used plants.

\section{INTRODUCTION}

Dandruff is a common scalp condition affecting majority of the population at pre-pubertal and post pubertal age, cutting across ethnicity, race and gender [1]. It is also believed to be caused by both microbial and non-microbial factors, with the most accepted etiological agent to be a lipophilic yeast that is of the genus Malassezia [2]. These etiologic agents are prevalent in regions with sebaceous glands because they are lipid dependent [3].

The Lipophilic dimorphic yeast Malassezia furfur is one of the common causative agents of dandruff including Malassezia restricta and Malassezia globosa. Malassezia furfur feeds on the dermal lipids and proteins which enhances and facilitates its lipase activity, causing inflammation and dermal tissue damage [2].

Dandruff cannot be completely eliminated, but can only be managed or controlled [4,5]. Eradication of dandruff causing fungus will facilitate effective treatment of dandruff leading to its management and effective control. Many substances available for the treatment of dandruff consist of various chemical based antifungal agents like Clotrimazole, Amphotericin B, Zinc pyrithione, Salicylic acid, Imidazole derivatives, Glycolic acid, Steroids, Sulfur, Tar derivatives and Nystatin etc. and are mostly associated with side effects such as dryness of hair/skin, associated cytostasis, and eczema, and also frequent reoccurrence made therapy costlier. So, it is imperative to search for drugs that are safe, cost-effective, and eco-friendly [6].

Plants rich in a wide array of antifungal secondary metabolites are gaining importance as a primary source of commercial medicines and drug leads, and have been used for the treatment of skin disorders for centuries $[7,8]$. Herbal drug technology has picked up strongly in terms of the extraction and characterization of active compounds and also the processing of herbs into medicine, considering the fact that modern medicine is not capable of providing a 
"cure all" solution for the pathogenic infections. Herbal therapy is considered as a therapeutic alternative, as a safer choice than synthetic medicines, or sometimes even as the only successful therapeutic way left to treat these disorders [6]. Therefore, this study was designed to isolate and determine the antifungal activities of Senna alata and Lawsonia inermis leaf extracts on Malassezia spp.

Phytochemical analyses revealed that Senna alata leaves contained saponins, alkaloids, flavonoids, tannins, phenols anthraquinione, protein and carbohydrate (glycosides) [9]. While Lawsonia inermis contain naphthalene derivatives, quinoids, $\beta$-sitosterol glycoside, xanthones, flavonoids, galic acid, coumarins and lawsoniasides. Lawsone, 2-hydroxy-1, 4naphthoquinone is responsible for henna's fungicidal activity.

\section{MATERIALS AND METHODS}

\subsection{Study Area}

This study was conducted at Auta-Balefi. Two sites were considered, Bingham University and Kodope primary school. Bingham University and Kodope Primary School are both located in AutaBalefi, along KM 26 Abuja Keffi Expressway Kodope, Karu, Nasarawa state. Bingham University alongside Kodope primary school covers a land mass of 200-220 square meters, it has a population of about 2000 students and is geographically located at latitude $8^{\circ} 50^{\circ} \mathrm{N}$ and longitude $7^{\circ} 52^{\circ} \mathrm{E}$. The University was established in the year 2005 [10]. Kodope Primary school is located at Bingham University Junction.

\subsection{Collection and Identification of Plant Samples}

Fresh leaves of the two plants used in this study were collected from Area one, Garki Abuja and Sayina village Auta-Balefi Karu LGA Nasarawa State. These leaves were collected separately into sterile polyethene bags and transported immediately to the laboratory for identification and processing. The plants were identified by expert in the department of Biological Sciences Bingham University Karu using herbarium key (ER135) for Senna alata (Cassia alata), and key (27544) for Lawsonia inermis. Characteristic features of the leaves such as shapes, sizes and flower such as the colour were used in the identification of plants. Other features include plant height, fruits they produce, nature of the branches, colour of the stems, present or absence of spines, and number of flowers.

\subsection{Preparation of Extracts}

The samples previously collected were dried at room temperature separately in the laboratory for one week. The dried leaves of Senna alata and Lawsonia inermis were further dried in a hot air oven separately to make grinding easier; they were grounded mildly into coarse powder using clean mortar and pestle after which it was transferred into a sterile bijou bottles and stored at $-20^{\circ}$ in a refrigerator before use [11].

\subsubsection{Ethanol extracts}

Twenty grams of the powdered material was extracted with $100 \mathrm{ml}$ of $80 \%$ ethanol, with constant agitation for 3 days and then filtered using a muslin cloth (Vandee and Peeranuch 2009).

\subsubsection{Aqueous extraction}

\subsubsection{Boiling}

Ten grams of each of the plant powdered samples were boiled in $100 \mathrm{ml}$ of water in separate beakers for 30 mins. The resulting extracts were filtered using Whatman No. 2 filter paper [12].

\subsubsection{Soaking}

Ten grams of the plant powdered sample was soaked in $100 \mathrm{ml}$ of distilled water for 24hours. The resulting extracts was allowed to cool down and then filtered using whatman No. 2 filter paper [12].

\subsection{Collection and Isolation of the Test Organisms}

Dandruff samples were obtained by scrapping the scalp surface with sterile combs. The combs used were sterilized by washing in detergent and soaking in $10 \%$ bleach solution to prevent contamination. Some portion of the scrapping from the scalp was inoculated over the surface of modified Dixion agar slants, transferred to the laboratory on the same day and inoculated at $30^{\circ} \mathrm{C}$ for 14 days. The morphology was examined on leeming and Notman agar after incubation at $32^{\circ} \mathrm{C}$ for 7 days [13]. 


\subsection{Identification of Fungus}

Identification of Malassezia was made microscopically by Loffler's Methylene Blue Staining and a drop of $10 \%$ potassium hydroxides $(\mathrm{KOH})$. Each slide was examined under high power field of microscope (40X objective) to reveal the presence of hyphae and budding yeast cells which generally exhibit the characteristic appearance of "spaghetti and meatballs" $[14,15]$.

\subsection{Biochemical Tests}

The gross morphology of suspected colonies of Malassezia which shows globose blastoconidia and mycelium was noted prior to biochemical test. Different biochemical tests such as the catalase test using hydrogen peroxide, bile Esculin splitting test, and Tween 20 and 80 utilization tests were carried out.

\subsubsection{Catalase test}

A small amount of fungal colony was transferred to a surface of a clean, dry glass slide using a wire loop, followed by a drop of hydrogen peroxide. Production of gas bubbles on adding a drop of hydrogen peroxide indicated a positive reaction.

\subsubsection{Bile esculin splitting test}

A fungal colony was streaked on bile Esculin agar and incubated at $37^{\circ} \mathrm{C}$ for $2-5$ days and development of black colored byproducts of Esculin hydrolysis indicates a positive test [16].

\subsubsection{Tween utilization tests}

A single colony from the 21 cultured sample plates was picked using a sterile cotton swap to make a lawn culture on the SDA plates and three wells were made using a borer on to which Tween 20 and 80 are inoculated. Utilization of Tween compounds was assessed by the degree of growth and or reaction (precipitation) of the lipophilic yeast around individual well [16].

\subsection{Evaluation of Antifungal Activity of Plant Extracts}

The antifungal effect of the plant extracts was evaluated by agar well diffusion method [14]. The surface of Sabouraud Dextrose Agar plates was inoculated with test microorganisms by spreading the surface of the media using sterile swabs.
Three wells were made using the borer with diameter $8.00 \mathrm{~mm}$ each for Senna alata and Lawsonia inermis extracts as well as the control (ethanol) to accommodate the three technical replicates. $10 \mu \mathrm{l}$ of the extracts was dispensed in the wells. The plates were incubated at $37^{\circ} \mathrm{C}$ for 2-5 days. The antifungal activity was determined by calculating the mean zones of inhibition of the 21 biological samples.

\section{RESULTS}

The overall prevalence of Malassezia spp. infection in the study area is $25.3 \%$ as shown in Table 1. The study also isolated three species as follows, Malassezia restricta 11(52.3\%), Malassezia furfur 5(23.8\%) and Malassezia globosa $5(23.8 \%)$, out of which Malassezia restricta was most prevalent. $M$. restricta was the main species causing dandruff, as it was isolated from $52 \%$ of the dandruff samples cultured; followed by $M$. furfur (24\%) and $M$. globosa (24\%) while $M$. pachydermatis and $M$. obtusa. Malassezia slooffiae and $M$. sympodialis were not found in any of the cases.

Table 2 shows the relationship of Malassezia spp. to gender of the students to be $7(33.3 \%)$ for males and $14(66.6 \%)$ for females $(P=0.367)$.

Table 3 shows higher occurrence among age group 21-25 (61.7\%) followed by age group 16$20(33.2 \%)$. M. restricta and M. globosa were not isolated among age group 10-15. M. furfur was found in all age group. The results did not demonstrate a significant relationship between the frequency of Malassezia species and age ( $p$ $=0.187)$, as well as gender $(P=0.367)$.

Table 4 shows the prevalence of Malassezia species with respect to institutions where samples were collected $(p=0.043)$. Out of the samples collected from Kodope primary school only one was positive for Malassezia furfur. $M$. restricta and $M$. globosa were not isolated from the primary school pupils. Samples collected from Bingham university students expressed all the three species isolated in the study.

Table 5 shows the mean zones of inhibition in millimetres and mean standard error (SEM) observed upon the application of aqueous extracts (soaked and boiled) and ethanolic extracts of $L$. inermis and $S$. alata, with ethanol serving as a control. The mean zone of inhibition for soaked extracts of $L$. inermis did not show any inhibitory effect but $S$. alata had inhibitory 
effects on the isolates. Also, both plants species extracted through boiling did not have any inhibitory effect. Ethanolic extract of $S$. alata had higher zone of inhibition of $11.00 \pm 0.969$ compared to the ethanolic extract of $L$. inermis (7.71 \pm 0.876$)$.

Table 1. The prevalence of Malassezia species in the study area

\begin{tabular}{lllll}
\hline Study locations & No. examined & No. positive & No. negative & Prevalence \\
\hline Kodape & 23 & 1 & 22 & $25.3 \%$ \\
Bingham & 60 & 20 & 40 & \\
Total & 83 & 21 & 62 & \\
\hline
\end{tabular}

Table 2. Malassezia species isolated in relation to gender

\begin{tabular}{llll}
\hline Species & Males & Females & Total \\
\hline Malassezia restricta & $5(23.8)$ & $6(28.5)$ & $11(52.3)$ \\
Malassezia furfur & $1(47)$ & $4(19)$ & $5(23.8)$ \\
Malassezia globosa & $1(4.7)$ & $4(19)$ & $5(23.8)$ \\
\hline
\end{tabular}

Table 3. Malassezia species in relation to age

\begin{tabular}{lllll}
\hline Organism & $\mathbf{1 0 - 1 5}$ & $\mathbf{1 6 - 2 0}$ & $\mathbf{2 1 - 2 5}$ & Total \\
\hline Malassezia restricta & $0(0)$ & $4(19)$ & $7(33.5)$ & $11(52.3)$ \\
Malassezia furfur & $1(4.7)$ & $1(4.7)$ & $3(14.2)$ & $5(23.8)$ \\
Malassezia globosa & $0(0)$ & $2(9.5)$ & $3(14.2)$ & $5(23.8)$ \\
\hline Total & $1(4.7)$ & $7(33.2)$ & $21(100)$ & $13(61.7)$ \\
\hline
\end{tabular}

Table 4. Malassezia species isolated in relation to institution

\begin{tabular}{llll}
\hline Organism & Kodape pri. sch. & Bingham university & Total \\
\hline M. restricta & $0(0)$ & $11(52.3)$ & $11(52.3)$ \\
M. furfur & $1(4.7)$ & $4(19)$ & $5(23.8)$ \\
M. globosa & $0(0)$ & $5(23.8)$ & $5(23.8)$ \\
\hline Total & 1 & 20 & 21 \\
\hline
\end{tabular}

Table 5. Mean standard error (SEM) of different extraction methods of (Senna alata and Lawsonia inermis) on Malassezia sp.

\begin{tabular}{lllll}
\hline & \multicolumn{3}{c}{ Methods of extractions } \\
\hline Plants sp. & Soaking & $\begin{array}{l}\text { Ethanolic } \\
\text { extracts }\end{array}$ & Control(Ethanol) & Boiling \\
\hline & Mean(SEM) & Mean(SEM) & Mean(SEM) & Mean(SEM) \\
\hline S. alata & $10.00 \pm 0.969$ & $11.00 \pm 0.969$ & $6.00 \pm 0.756$ & $0.00 \pm 0.00$ \\
L. inermis & $0.00 \pm 0.00$ & $07.71 \pm 0.876$ & $4.000 \pm 0.396$ & $0.00 \pm 0.00$ \\
\hline
\end{tabular}

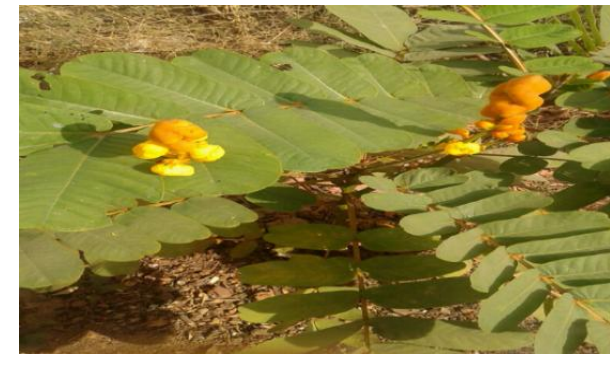

Plate 1. Senna alata

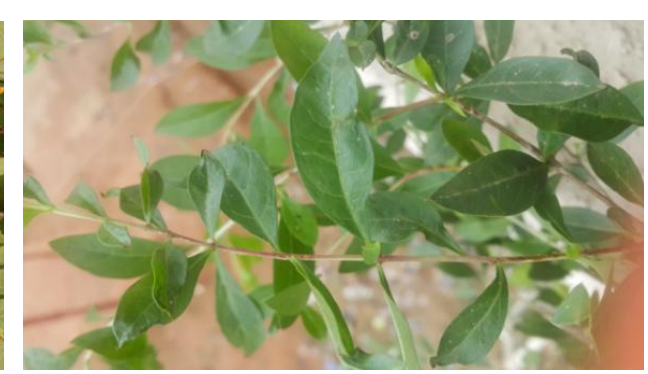

Plate 2. Lawsonia inermis 


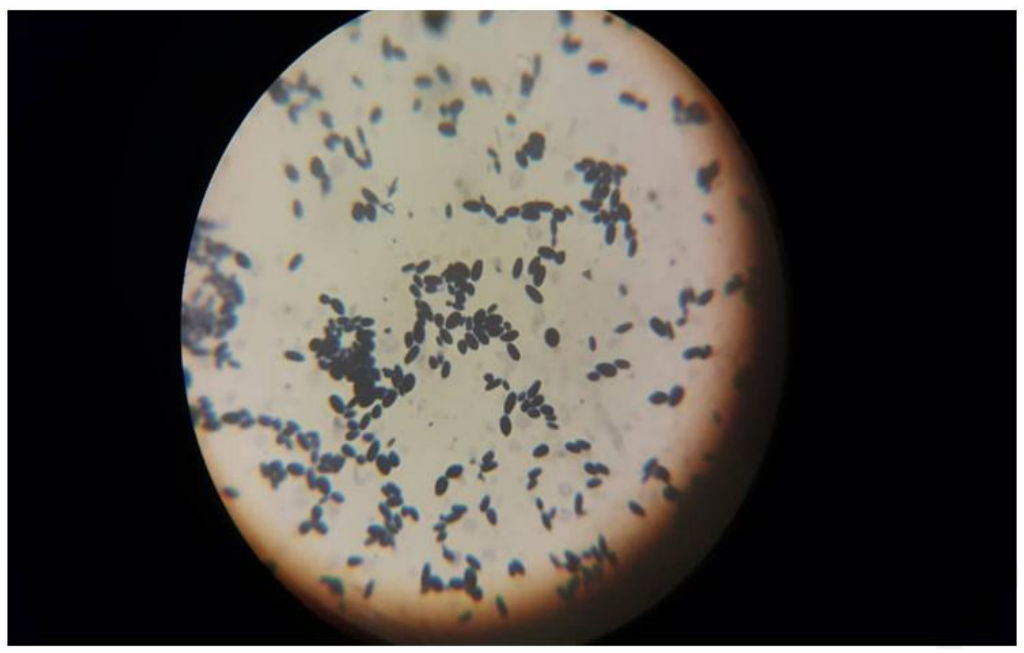

Plate 3. Culture of Malassezia sp.

\section{DISCUSSION}

Malassezia species are yeasts that make up part of the microbiota of the mammalian skin. They are prevalent in regions with sebaceous glands due to their dependency on lipids [3]. This study revealed $25.3 \%$ prevalence of Malassezia species. The low level of infection recorded in this study could be due to use of other synthetic antifungal creams by the subjects. This prevalence is low compared to the findings of Hedayati et al. [17] and Zareei et al. [18] both of whom worked independently and reported higher prevalence of $70 \%$ and $93.5 \%$ respectively in their studies.

Three species that characterised this study were $M$. restricta, $M$. furfur and $M$. globosa. Out of the three species encountered in this study, $M$. restricta was the most common species followed by $M$. furfur and M. globosa. Other similar studies who reported these species were the reports of Shivaprakas et al. [19], Shu'aibu et al. [14], and Zareei et al. [18]. However, their findings differ slightly with this finding in that they all reported different species to be the most prevalent. This is evident in the reports of Zareei et al. [18] who reported $M$. globosa as the most significant species while $M$. sympodialis $(6.5 \%)$ and $M$. slooffiae $(0.8 \%)$ were the least fungal species isolated. Hedayati et al. [17] reported the $M$. globosa as the most commonly isolated species followed by $M$. furfur and $M$. restricta. Shu'aibu et al. [14] also reported $M$. globosa as the most predominant species. These variations may be due to the fact that Malassezia species implicated with dandruff vary at different geographical locations, study participants or isolation method used.

Higher infection was recorded among University students with all the three species isolated from the students. This finding disagrees with the finding by Shivaprakash et al. [19] who recorded higher prevalence among age group 10-19. This is so because other studies indicated differences in the colonisation pattern of Malassezia species with different age groups with higher colony counts in adults than in children and a decrease in the elderly individual [20]. This condition may be due to high sebum production in the abovementioned age groups, microbial metabolism or susceptibility of the individual.

This study evaluated the anti-malassezia effects of the two plants species $S$. alata and $L$. inermis. The result reveals $S$. alata to exhibit some antimalassezia effect on the isolates with mean zone of inhibitions of $11 \pm 0.969$ for ethanolic extracts and $10 \pm 0.969$ for soaked (aqueous) extracts. From these findings and other reports from Borade et al. [7], Jung et al. [21] and Wandee and Peeranuch [11], the ethanolic extracts seem to exhibit higher zone of inhibition than the aqueous solution. This may not be far from the reports of Natarajan et al. (2005) that different solvents have different solubility capacities for different phyto-constituents, thus, the differences in the activities of the various extracts [20,21,22].

In the case of $L$. inermis plant extract, the result shows lower anti-malassezia effect compared to S. alata with no zone of inhibition for soaked and boiled extracts. Only the Ethanolic extracts of 
$L$. inermis shows some inhibitory activities on the isolate which is $7.71 \pm 0.876$.

\section{CONCLUSION}

This study reveals that naturally acquired herbs of $S$. alata especially and $L$. inermis are effective in combating Malassezia species which are the causative agents of dandruff and hence, the use of naturally acquired herbs should be encouraged in combating dandruff.

\section{CONSENT AND ETHICAL APPROVAL}

Ethical approval to conduct research was sought from Nasarawa State Hospital Management Broad (NSHMB) through the Research Ethics Committee of Nasarawa State Ministry of Health Lafia, in line with the guidelines required for conducting research on human samples. Further Approval was sought from the respective school management as well as Informed consent from the volunteers and their parents / guardians.

\section{COMPETING INTERESTS}

Authors have declared that no competing interests exist.

\section{REFERENCES}

1. Ranganathan S, Mukhopadhyay $\mathrm{T}$. Dandruff: The most commercially exploited skin disease Indian Journal of Dermatology. 2010;55(2):130-134. DOI: 10.4103/0019-5154.62734

2. Rafiq S, Ahmed NSK, Shahina J. Isolation and characterization of fungi from dandruffafflicted human scalp and evaluation of Anti-dandruff shampoo. Indian Journal of Applied Research. 2014;253.

DOI: $10.36106 /$ IJAR

3. Arora PDN. Screening of plants essentials oils for antifungal activities against Malassezia furfur. International Journal of Pharmacy and Pharmaceutical Science. 2012;37-39.

4. Kumar PV, Venkateswara P, Prince R, Terejamma R, Chaitanya K, Prasanna TKD. Formulation and evaluation of herbal anti-dandruff shampoo from bhringraj leaves. ARC Journal of Pharmaceutical Sciences (AMPS). 2018;4(2);29-33.

ISSN No: 2455-1538.

DOI:http://dx.doi.org/10.20431/24551538.0402005
5. Arun PPK. In vitro antibacterial activities and flavonoid contents of Lawsonia inermis (henna). International Journal of Pharmaceutical and Technological Research. 2010;1178-1181.

6. Sreelatha GL, Lakshmeesha TR, Jayashree B, Sharmila T, Soumya K. insignificant antifungal activity of plant extracts on Malassezia furfur. 2016; 7(1):16-20.

DOI: $10.4103 / 0976-9234.177055$

7. Borade AS, Babasaheb N. Kale, Rajkumar VSA. A phyto-pharmacological review on Lawsonia inermis Linn. International Journal of Pharmaceuticals and Life Science. 2011;536-541.

ISSN: 0976-7126

8. Lalitha SG, Ramachandrappa LT, Krishnamurthy S, Basavaraju J; Thirumale S. Insignificance of Antifungal activity of plant extracts on $M$. furfur Journal of Pharmaceutical Negative Results. Published by Wolters Kluwer - Medknow; 2016.

9. Karthika CK, Rafi MK, Manivannan S. phytochemical analysis and evaluation of anti-microbial potentials of Senna alata linn. Leaves extracts. Asian Journal of Pharmaceutical and Clinical Research. $9^{\text {th }}$ Edition; 2016.

10. Bingham University; 2017.

Available:https://www.binghamuni.edu.ng/a boutus.php

Available:https://www.binghamuni.edu.ng/a boutus.php

11. Wandee G, Peeranuch M. Standardized Senna Alata Leaf Extract. Journal of Health Research. 2009;59-64.

12. Azwanida NN. A review on the extraction methods use in medicinal plants, principle, strength and limitation. Medicinal and Aromatic Plants. 2015;4:3.

DOI: $10.4172 / 2167-0412.100019$

13. Ashtiani HA, Rastaga $H$, Mamoud $A$, Amirhushang $E$, Behrooz B, Mina HS. Clinical efficacy of natural formulated shampoo in subjects with dandruff and seborrheic dermatitis. American Journal of Research Communication. 2013;1(8): 63-80.

ISSN: 2325-4076

14. Shu'aibu I, SA'ad H, Umar FM, Maikudi MM. Isolation and identification of Malassezia globosa, associated with dandruff among female students of Gombe state university. Greener Journal of 
Microbiology and Antimicrobials. 2013; 1(1):001-006.

ISSN: 2354-2284.

15. Surabhi $P$, Vaishali $M$. Studies on antifungal activities of certain plant extracts against dandruff-causing fungus, Malassezia. International Journal of Current Research in Biosciences and Plant Biology. 2015;2(7):206-211.

16. Elham Z, Golnar S, Fehimeh $\mathrm{Y}$, Masoomeh SG, Mehdi RA. Clinical and epidemiological features of genus Malassezia in Iran. Iranian Journal of Microbiology. 2014;6(5):354360.

17. Hedayati MT, Hajheydari Z, Hajjar F, Ehsani A, Shokohi T, Mohammad PR. Identification of Malassezia species isolated from Iranian seborrhoeic dermatitis patients. European Review for Medical and Pharmacological Sciences. 2010;14:63-68.
18. Zareei M, Mohammad A, Borjian Z, Seyed $\mathrm{BI}$, Hashemi Jl. Frequency of Different Malassezia species in scalp dandruff. Infection, Epidemiology and Medicine. 2016;2(2):22-25.

DOI: 10.7508/iem.2016.02.007

19. Shivaprakash M. Rudramurthy $\mathrm{PH}$. Association of Malassezia Species with Dandruff. Indian Journal of Medical Research. 2014; 139:431-437.

20. Gupta AK, Kohli Y, Summerbell RC, Faergemann J. Quantitative culture of Malassezia species from different body sites of individuals with or without dermatoses. Med. Mycol. 2001;39;243251.

21. Jung EKY. Physicochemical properties and antimicrobial activity of Roselle (Hibiscus sabdariffa $L$. Journal of food Science and Agriculture. 2013;93:3769-3776.

22. Natarajan V. Antifungal properties of Neem. Indian Journal of Medical Microbiology. 2003;98-101.

(c) 2021 Ishaku et al.; This is an Open Access article distributed under the terms of the Creative Commons Attribution License (http://creativecommons.org/licenses/by/4.0), which permits unrestricted use, distribution, and reproduction in any medium, provided the original work is properly cited.

Peer-review history:

The peer review history for this paper can be accessed here: http://www.sdiarticle4.com/review-history/65464 